\title{
Follicular Neoplasm Unmasking Ectopic Thyroid
}

\author{
Dr.AshishGopal*Dr. Sangeeta Singh**,Dr. Meenakshi Sridhar*** \\ 1.(Department Of E.N.T/Aruna Asaf Ali Govt. Hospital, New Delhi, India.)* \\ 2. (Department Of E.N.T/Aruna Asaf Ali Govt. Hospital, New Delhi, India.)** \\ 3.(Department Of PATHOLOGY/Dr.Baba Saheb AmbedkarHospital,New Delhi, India).***
}

\begin{abstract}
Rare possibilities like an adenoma or a malignancy may occur in ectopic thyroid tissue. These pathologies can mimic many midline or para-midline neck masses.Such conditions require a good clinical history and examination, apart from a keen eye for suspicion.

We report a case of 35 year female presenting with a midline neck lump for 14 months.Elsewhere patient was worked up for suspicion of thyroglossal cyst and a tubercular node. We performed preliminary investigations including Thyroid profile, Fine Needle Aspiration Cytology (FNAC) and ultrasonography(USG) neck followed by Thyroid Sctintigraphy and Magnetic.Resonance Imaging (MRI) neck to confirm an ectopic thyroid and a satisfactory diagnosis of ectopic thyroid tissue undergone adenomatous transformation was made. Surgical neck exploration for removal of adenoma and pyramidal lobe with isthmusectomy was performed. Surgical specimen rendered for histopathology examination(HPE), confirmed the diagnosis. Clinical intuition and suspicion can unmask a rare pathology, not seen on day to day basis.
\end{abstract}

Key Words: EctopicThyroid, Follicular Adenoma, Midline Neck mass, Thyroid

\section{Introduction}

Occurrence of rare possibilities such that of an ectopic thyroid which can mimic a bewildering array of midline and para-midline neck masses that have to be excluded with keen examination. A developmental anomaly such that of an undescended thyroid tissue or ectopic thyroid may lay hidden and get revealed by a pathology occurring later in life, is a rare phenomenon. This pathology either benign or malignant can unmask part or full tissue of the orthopic thyroid lying anywhere from the foramen caecum till the actual site of normal thyroid in the neck. Clinical suspicion of such a condition should be borne in mind to institute needful investigations and definitive treatment.

\section{II.Case Report}

A 35 year old lady presented to us with a midline neck swelling for duration of 14 months. The swelling was $4 \times 4 \mathrm{~cm}$, firm in consistency, moving on deglutition and present at the level of hyoid. The differential diagnosis of enlarged lymph node, thyroglossal cyst and that of ectopic thyroid was kept in mind, other than that of a tubercular node. Euthyroid state was confirmed by thyroid function test. The USG neck showed a homogenous lesion comprising with echo-texture alike with the rest of the normal, eutopic thyroid. The F.N.A.C. showed high cellularity of follicular cells with repetitive micro-follicular pattern and focal areas of nuclear overlapping. A study of pertechnetate thyroid flow scan showed poor tracer uptake in the lesion. Yet for further certainty and to confirm the nature and extent of the lesion, M.R.I was done. MRI described the lesion to be abutting from antero - superior region of right isthmus with possible presence of a pyramidal lobe. The lesion appeared hyper - intense on $\mathrm{T} 1 \mathrm{~W}$ and $\mathrm{T} 2 \mathrm{~W}$ and fat suppressed images, at the same time confirmed the presence of a normal sized and normal signal intensity thyroid lower in the neck. A diagnosis of ectopic thyroid tissue with adenomatous transformation was ascertained. The patient was operated with a standard neck incision providing good visualization and surgical manipulation. A normal thyroid was first confirmed in the neck which was giving rise a pyramidal lobe and superiorly it expanded into a $4 \times 4 \mathrm{~cm}$ round mass in infra-hyoid region. The pulsatile presence of a thyroid Ima artery, lying adjacent to the pyramidal lobe was an added finding .We performed an isthmusectomy with removal of pyramidal lobe and the adenomatous circumferential lesion along with the ligation of the Ima artery. A meticulous dissection showed a thin fibrous linear band of tissue regressing deep to the adenomatous ectopic mass below the hyoid. The histopathology report showed an encapsulated follicular adenoma in the ectopic gland. Macroscopically measuring $4 \times 4 \mathrm{~cm}$, Light microscopy revealed a micro follicular and trabecular growth pattern and attenuated normal thyroid parenchyma in the immediate vicinity of the capsule, there was no capsular or vascular invasion. The patient was on regular follow up for 18 months showed no recurrence. 


\section{III.Discussion}

Ectopic thyroid is a rare clinical entity with incidence of $1 / 3000$ to $1 / 10,000$ in general population [1] , resulting from developmental defects at early stages of thyroid gland embryogenesis, during its passage from the floor of the primitive foregut to its final pre-tracheal position. It was first described by Hickman in 1869. A short literature on ectopic thyroid, is as follows. Thyroid gland is the first endocrine gland to develop during embryogenesis on approximatelythe 24th day of gestation. It arises after proliferation of endodermal epithelial cells in the pharyngeal floor, called foramen caecum. A diverticulum invaginates from the foramen cecum , proceeds deep down into the neck to bifurcate into two lobes of the thyroid. It is the arrest of this median thyroid anlage or diverticulum between the $3^{\text {rd }}$ and $7^{\text {th }}$ week of gestation that results in thyroid ectopia [2] . This undescended tissue of the thyroid commonly presents as lingual thyroid in $90 \%$ of all cases .Other sites of ectopic thyroid are mediastinum (arch of aorta, trachea and heart), around the common bile duct, retroperitoneal, vagina, ovaries, inguinal region and sellaturcica.

Follicular adenoma of thyroid arising in thyroid ectopia has rarely been reported in literature. The incidence of ectopic thyroid tissue turning into goitrous enlargement either benign adenoma or a malignant neoplasm is same as in normal thyroid. The etiology of thyroid adenoma is obscure, but an initial adenomatous change is probably a result of TSH priming. They can occur at any age and usually present as solitary nodules.Ectopic thyroid occurs 3-4 times more commonly in females and can occur in any age group. These adenomas are encapsulated, with occasional cystic areas and are microscopically distinguished into varieties as trabecular, fetal, colloidal or Hurthle cell types. Pathological evidence indicates a rare transformation of adenoma into invasive carcinoma. Further genetically mutation of ras gene has been confirmed with thyroid adenomas. We followed a protocol of these investigations, thyroid profile, F.N.A.C., ultrasound neck, thyroid scintigraphy and M.R.I. The needle cytological examination is viewed as the gold standard, it provides adequate tissue in $90 \%$ cases and has a histological accuracy near or superior to $95 \%$ [3] Ultrasonography is a more specific investigation in these cases in order to delineate size, nature of lesion, and tumors $3 \mathrm{~mm}$ or more U.S.G. is of more help, smaller than $3 \mathrm{~mm}$ are a frequent finding in thyroid on U.S.G. and are referred to as "incidentalomas". Scintigraphy using radioiodine or pertechnetate reveals location and functional volume of thyroid tissue. Technecium $99 \mathrm{~m}$ in the form of pertechnetate is relatively inexpensive, gives lower radiation exposure, does not undergo organification and less scan time is possible, 15 to 30 minutes after injection. Nearly $70 \%$ thyroid adenomas are hypo functional on sctintigraphy and show cold nodule; $20 \%$ border up with same uptake as normal thyroid tissue. The remainder avidly concentrates the radio-active material and is hyperfunctional [4] .M.R.I. is indicative for better tissue differentiation and is free from radiation, further non iodinated contrast medium used scores better than C.T.scan. In general M.R.I. in these lesions shows a more tense signal on T1 weighted images. However, still U.S.G. lends better information and is more dependable than M.R.I. in thyroid lesions.

\section{Figures}

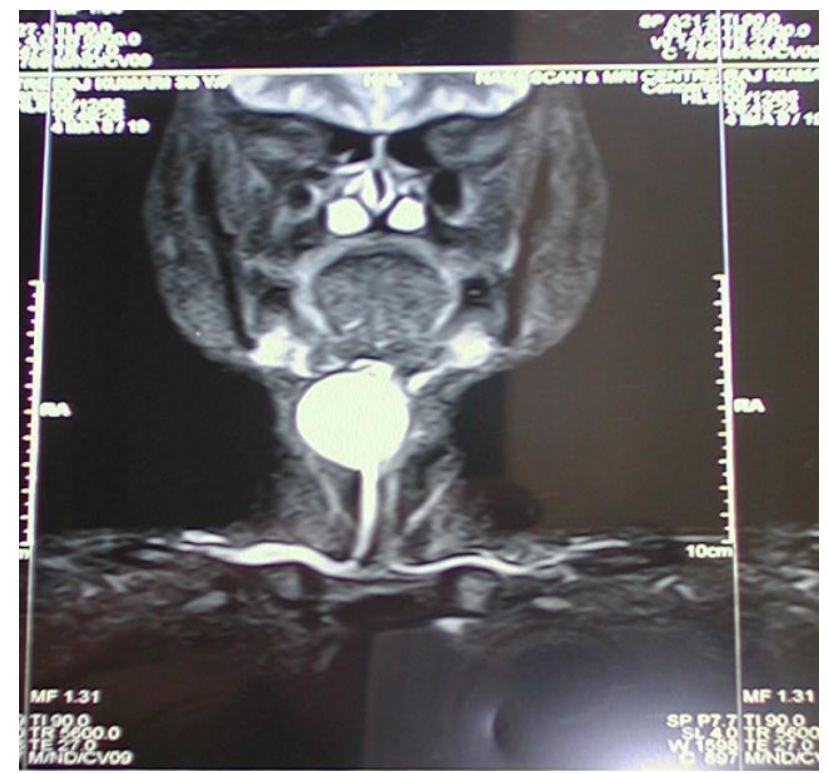

Figure no. 1:M.R.I. of the neck showing adenoma with attached pyrimidal lobe abbutting from right thyroid gland. 


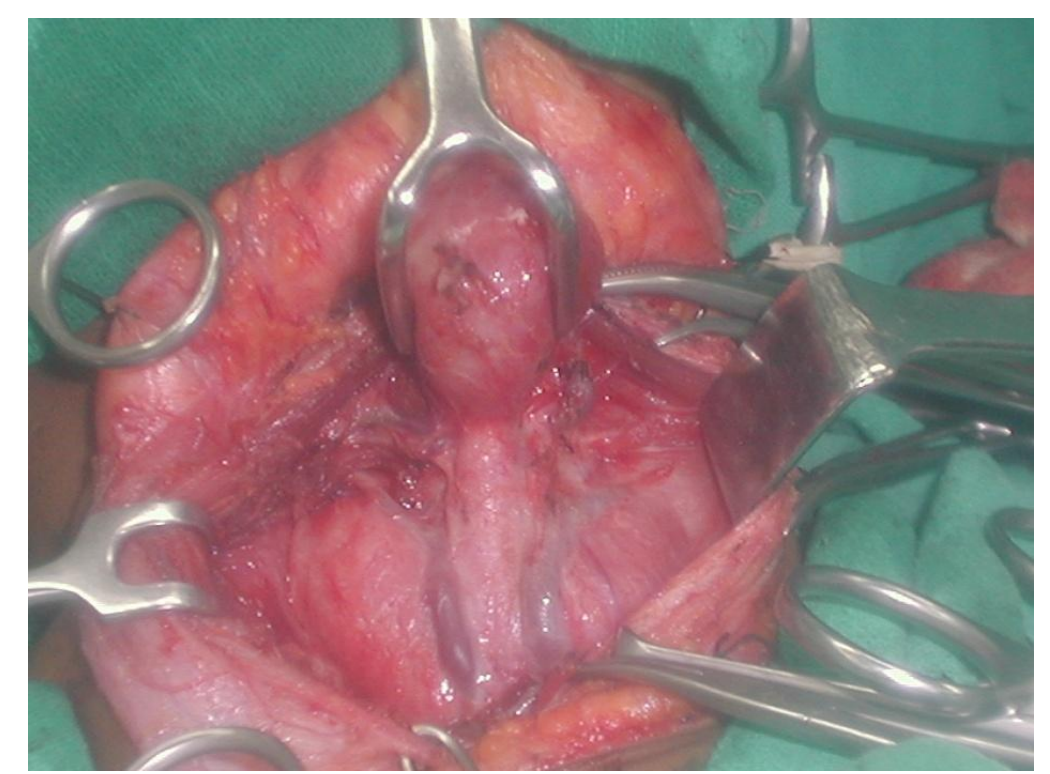

Figure no. 2: Picture of the neck after exploration showing the normal thyroid gland lobes and pyrimidal lobe from adenoma descending to right end of isthmus.

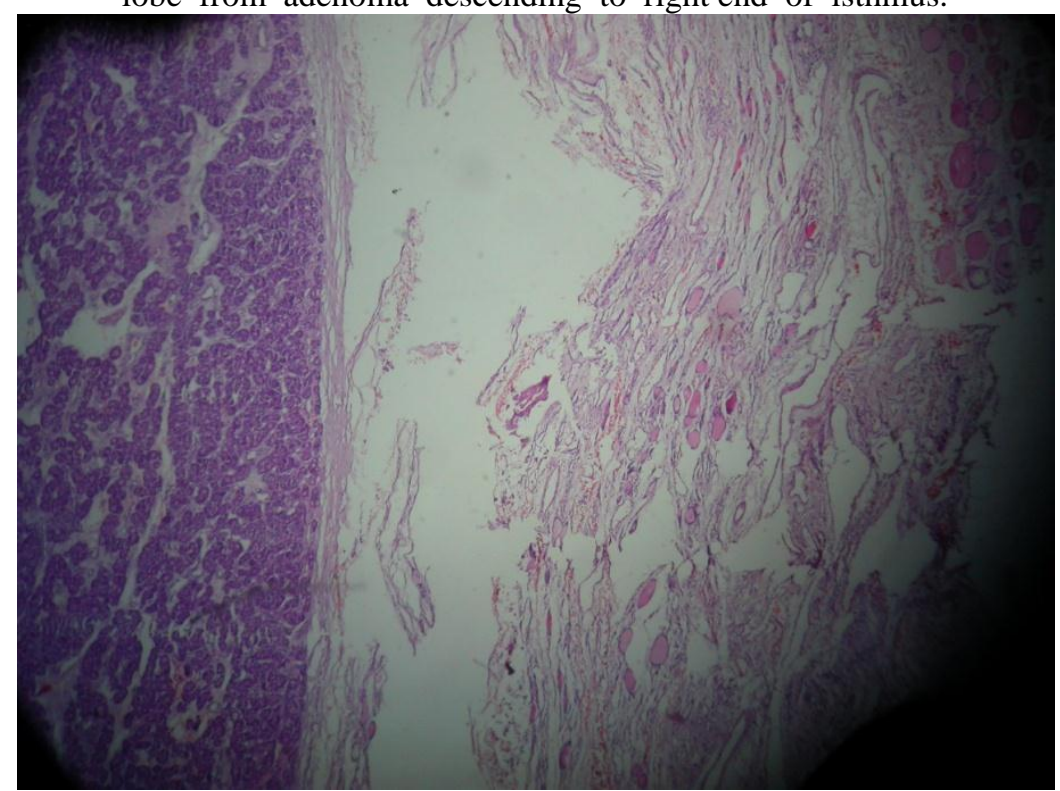

Figure no.3:Histopathology showing follicular cells in a repetitive micro-follicular pattern and bordering normal thyroid tissue.(MGG x 10)

\section{Conclusion}

Midline or Para-midline pathologies such that of tubercular node, thyroglossal cyst, salivary gland related adenomas or mucoceles, nerve sheath tumors and metastatic diseases often need to be distinguished as other diagnosis. In conclusion all midline and lateral neck swellings require a comprehensive study keeping in mind a rare, possible reality of it being an ectopic thyroid and to be investigated in the lines of the above pertinent investigations.

\section{Acknowledgements}

We thank and acknowledge the functionaries of our hospital who aided us and the patient and relatives in this above mentioned case report 


\section{References}

[1] H.Sargin, G.Kobacam, O. Köseoğulları, A. Şanlı, F. Murat, Erdoğan ,Three cases of lingual thyroid and review of literature.Turkish Journal of Endocrinology and Metabolism,3, 01/2003, 131-135.

[2] A.SosciaG.Guerra, MP .Cinelli, D .Testa, V Galli, V Macchi, R . De Caro,Para pharyngeal ectopic thyroid: The possible persistent of the lateral thyroid anlage -Clinical case report. Surgical and Radiological Anatomy, Aug; 26(4) 2004, 338-43. Epub 2004 May 8.

[3] H. Gharib, J.R.Goellner ,D.A. Johnson . Fine needle aspiration cytology of the thyroid: A 12 year experience with 11,000 biopsies. Clinical Laboratory Medicine Sep;13,1993, 699-709.

[4]. Leslie j De Groot, J.Larry Jameson .Endocrinology adult and pediatric -thyroid gland(Elsevier Health Science,Engelska ) 2013$05-0$ 